\title{
Interarm Blood Pressure Difference has Various Associations with the Presence and Burden of Cerebral Small-Vessel Diseases in Noncardioembolic Stroke Patients
}

\author{
Yoonkyung Chang ${ }^{\mathrm{a}, \mathrm{b}}$ \\ Seung Ah Lee ${ }^{a}$ \\ Sue Hyun Lee ${ }^{a}$ \\ Eun Hye Lee ${ }^{a}$ \\ Yong-Jae Kim ${ }^{\mathrm{a}}$ \\ Tae-Jin Song ${ }^{\mathrm{a}}$ \\ ${ }^{a}$ Department of Neurology, \\ College of Medicine, \\ Ewha Womans University, Seoul, Korea \\ ${ }^{\mathrm{b}}$ Departent of Neurology, \\ College of Medicine, Korea University \\ Guro Hostpital, Seoul, Korea
}

\begin{abstract}
Background and Purpose An interarm blood pressure difference (IABD) is independently related to the occurrence of cardiovascular disease and mortality. Cerebral small-vessel diseases (SVDs) are important risk factors for stroke, cognitive dysfunction, and mortality. We aimed to determine whether IABD is related to cerebral SVDs.

Methods This study included 1,205 consecutive noncardioembolic ischemic stroke patients as confirmed by brain MRI and simultaneously measured the bilateral brachial blood pressures. We investigated cerebral SVDs based on high-grade white-matter hyperintensities (HWHs), presence of cerebral microbleeds (CMBs), high-grade perivascular spaces (HPVSs), and asymptomatic lacunar infarctions (ALIs) on brain MRI.

Results In multivariate logistic regression, an interarm systolic blood pressure difference (IASBD) $\geq 10 \mathrm{~mm} \mathrm{Hg}$ was independently related to the existence of HWHs [odds ratio $(\mathrm{OR})=$ $1.94,95 \% \mathrm{CI}=1.32-2.84, p=0.011]$ and had a tendency to be associated with the presence of HPVSs $(\mathrm{OR}=1.45,95 \% \mathrm{CI}=0.49-2.23, p=0.089)$ and ALIs $(\mathrm{OR}=1.42,95 \% \mathrm{CI}=0.96-2.11, p=$ $0.052)$, but not with the presence of $\mathrm{CMBs}(\mathrm{OR}=1.09,95 \% \mathrm{CI}=0.73-1.61, p=0.634)$. In multivariate linear regression adjusted for age, sex, and variables with $p<0.1$ in the univariate analysis, IASBD $\geq 10 \mathrm{~mm} \mathrm{Hg}$ and interarm diastolic blood pressure difference $\geq 10 \mathrm{~mm} \mathrm{Hg}$ were significantly correlated with an increased total burden of SVDs $(\beta=0.080$ and $p=0.006$, and $\beta=0.065$ and $p=0.023$, respectively).

Conclusions This study found that IABD $\geq 10 \mathrm{~mm} \mathrm{Hg}$ was associated with the presence and increased burden of cerebral SVDs in noncardioembolic stroke patients. This suggests that IABD $\geq 10 \mathrm{~mm} \mathrm{Hg}$ could be a useful indicator of the presence and burden of cerebral SVDs in stroke patients.
\end{abstract}

Key Words asymptomatic lacunar infarctions, cerebral microbleeds, cerebral small-vessel diseases, interarm blood pressure difference, white-matter hyperintensities.
Received August 23, 2018

Revised October 22, 2018

Accepted October 22, 2018

\section{Correspondence}

Tae-Jin Song, MD, PhD

Department of Neurology,

College of Medicine,

Ewha Womans University,

1071 Anyangcheon-ro, Yangcheon-gu,

Seoul 07985, Korea

Tel +82-2-2650-2677

Fax +82-2-2650-5958

E-mail knstar@ewha.ac.kr

\section{INTRODUCTION}

An interarm blood pressure difference (IABD) is frequently observed clinically. The prevalence of IABD $\geq 10 \mathrm{~mm} \mathrm{Hg}$ is $4.4 \%$ in the general population without vascular disease, but this is higher in patients with cardiovascular risk factors including diabetes mellitus, hypertension, and stroke. ${ }^{1,2}$ The IABD was reported to be independently related to cardiovascular and all-cause mortality, ${ }^{3}$ and this association has been demonstrated in cohorts without pre-existing cardiovascular disease. ${ }^{4}$

Cerebral small-vessel diseases (SVDs) represent ischemic or hemorrhagic damage in cerebral small arteries that appear as white-matter hyperintensities (WMHs), cerebral microbleeds (CMBs), perivascular spaces (PVSs), and asymptomatic lacunar infarctions (ALIs)

(a) This is an Open Access article distributed under the terms of the Creative Commons Attribution Non-Commercial License (https://creativecommons.org/licenses/by-nc/4.0) which permits unrestricted non-commercial use, distribution, and reproduction in any medium, provided the original work is properly cited. 
in brain MRI. 5,6 Although each SVD has diverse implications (e.g., WMHs, PVSs, ALIs for cerebral ischemia, and CMBs for cerebral hemorrhage), all cerebral SVDs have similar vascular risk factors or pathogenic mechanisms. ${ }^{7.8}$ Because these cerebral SVDs are independently associated with cognitive dysfunction, future stroke, and the prognosis, ${ }^{5,69,10}$ it is important to identify the factor associated with cerebral SVDs in order to prevent and treat these neurological diseases.

The IABD may be due to stenosis or stiffness of the subclavian artery or aorta, and it may induce cerebral hypoperfusion that results in brain damage. ${ }^{11}$ Moreover, IABD is associated with elevated arterial stiffness in the elderly. ${ }^{12}$ Because cerebral hypoperfusion and arterial stiffness are closely associated with cerebral SVDs, IABD may be related to the presence and burden of cerebral SVDs. However, few studies have attempted to confirm this association. We therefore aimed to determine that whether IABD is related to cerebral SVDs.

\section{METHODS}

\section{Subjects}

Between January 2012 and June 2016, 1,958 consecutive patients who developed first-ever acute stroke within 7 days after developing neurological symptoms were included from our prospective stroke registry. ${ }^{13}$ During admission, demographics, past medical and medication history, clinical and neurological presentation, and classical risk factors for cerebrovascular disease were registered. Based on the protocol of our department, brain CT and/or MRI, imaging of the cerebral vasculature, chest X-rays, 12-lead cardiac electrocardiography, and blood laboratory tests were routinely performed. For investigating concomitant systemic atherosclerosis, the ankle-brachial index (ABI) examination was also routinely performed during admission using an automated device (VP-1000, Colin Medical Technology Corporation, Komaki, Japan). ${ }^{13,14}$

These 1,958 subjects did not include patients with a potential source of cardioembolism [ $n=301$; atrial fibrillation $(n=$ 270 ), sick sinus syndrome $(n=5)$, and other cardioembolic source $(n=26)$ ], rare causes such as dissection or venous thromboembolism $(n=45)$, transient ischemic attack ( $n=$ 205), or undetermined etiology due to an incomplete investigation ( $n=17)$. Because arrhythmia could prohibit the accurate investigation of arterial stiffness [brachial-ankle pulse wave velocity (baPWV)] and blood pressure, patients with cardioembolism stroke subtype were excluded, ${ }^{2,15}$ as were patients in whom brain MRI was not performed $(n=25)$, images of the cerebral intra- and extravasculature were not available ( $n=12$ ), gradient recalled echo (GRE) images were not available $(n=8)$, and the available MRI images were of poor quality $(n=6)$. Additionally, subjects in whom the ABI examination was not performed $(n=72)$ or $\mathrm{ABI}<0.9(n=62)$, which may be related to inaccurate measurements of the arterial stiffness and blood pressure ${ }^{16}$ were also excluded. Ultimately 1,205 patients were finally included in our study (Fig. 1). The stroke classification was determined based on the Trial of Org 10,172 in Acute Stroke Treatment classification system. ${ }^{17}$ Our study was approved by the Institutional Review Board of our hospital (IRB No. 2017-04-017-001), and the requirement to obtain informed consent from patients was waived because of the retrospective, cross-sectional, and observational design of the study.

\section{Measurement of blood pressures in both arms and IABDs}

The systolic and diastolic blood pressures were investigated in both arms with the subject in a supine position using an automated device designed primarily to measure ABI. The ABI test was performed by a well-trained examiner with more than 5 years of experience after the subject had rested in a quiet room for at least 5 minutes. The blood pressure was measured bilaterally in the brachial and posterior tibial arteries automatically and simultaneously using the oscillometric method. ${ }^{18}$ These blood pressures in both arms were checked once. Blood pressure differences in the lower limbs were not used in our study because such evaluations were outside the scope of this study. The presence of a significant IABD [interarm systolic blood pressure difference (IASBD) and/or inter-

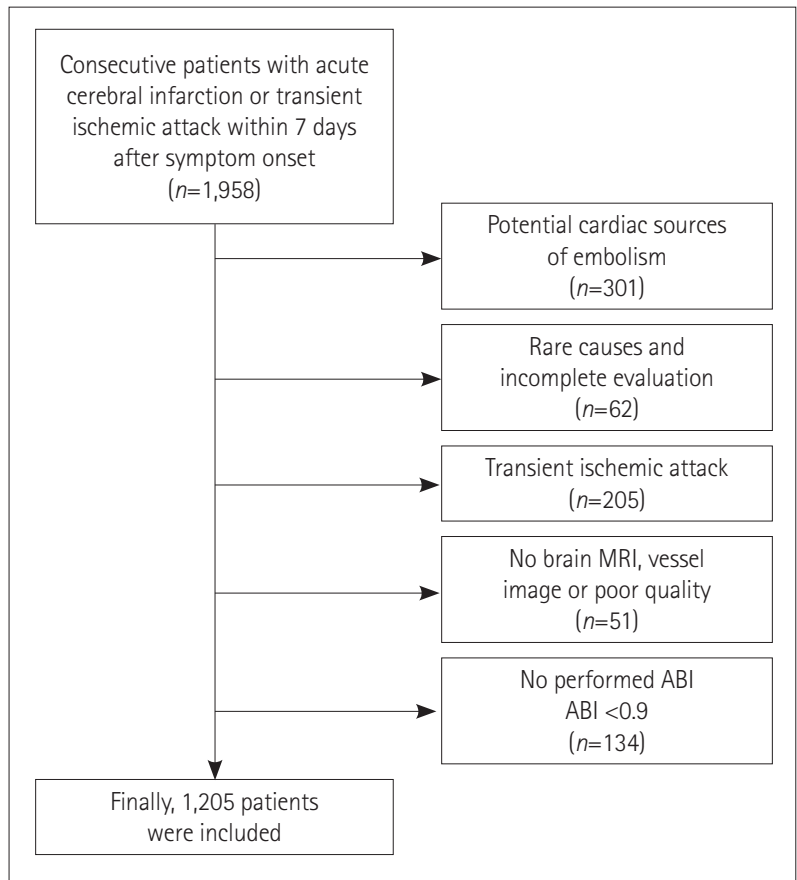

Fig. 1. Flowchart of participants according to the applied inclusion and exclusion criteria. $\mathrm{ABI}$ : ankle-brachial index. 
were blinded to the clinical information.

The total SVD score was determined as the summation of all cerebral SVDs present. One point was given for each of the following parameters: existence of HWHs, CMBs, HPVSs, or ALIs. ${ }^{24}$ The coefficients for the interobserver agreements on the existence of HWHs, CMBs, HPVSs, and ALIs were 0.912, $0.956,0.938$, and 0.888 , respectively. If there was disagreement over the existence of cerebral SVDs, a final decision was reached by consensus.

\section{Clinical and laboratory variables}

The risk factors for hypertension, diabetes mellitus, hyperlipidemia, smoking, coronary artery disease, metabolic syndrome, and alcohol intake were defined in detail in a previous study ${ }^{25}$ and Supplementary Material (in the online-only Data Supplement). Antihypertensive treatment after admission was defined as treatment with intravenous or oral antihypertensive agents within 7 days of admission. Our target level of antihypertensive therapy was defined as a systolic blood pressure of up to 200-220 mm Hg or a diastolic blood pressure of 120 $\mathrm{mm}$ Hg. However, in patients receiving thrombolytic treatment, the target level of antihypertensive therapy was a systolic blood pressure of up to $185 \mathrm{~mm} \mathrm{Hg}$ or a diastolic blood pressure of $110 \mathrm{~mm} \mathrm{Hg}$. Left ventricular hypertrophy was diagnosed when electrocardiography findings were matched with at least one of the relevant voltage criteria. ${ }^{26}$ The baPWV was defined as the mean baPWV value bilaterally.

\section{Statistical analysis}

The Windows SPSS software package (version 21.0, IBM Corp., Armonk, NY, USA) was used for statistical analysis. Categorical variables are expressed as frequency and percentage values, and continuous variables are expressed as mean $\pm S D$ values. Differences in demographic characteristics, presence of vascular risk factors, and brain MRI findings were compared between patients with IASBD and IADBD using the chi-square test, Fisher's exact test, and the independent $t$-test.
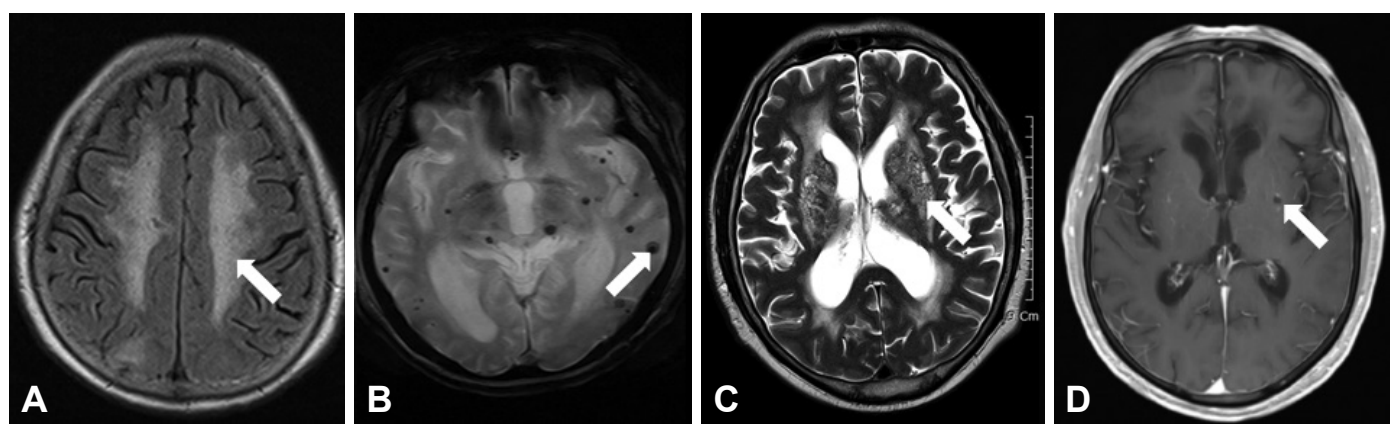

Fig. 2. Examples of cerebral small-vessel diseases. The arrows indicate high-grade white-matter hyperintensities (A), cerebral microbleeds (B), highgrade perivascular spaces (C), and an asymptomatic lacunar infarctions (D). 
Receiver operating characteristic (ROC) curves were investigated in terms of the area under the curve (AUC), standard error, $p$ value, sensitivity, and specificity according to $\mathrm{IASBD} \geq 10 \mathrm{~mm} \mathrm{Hg}$ or IADBD $\geq 10 \mathrm{~mm} \mathrm{Hg}$.

The associations between the presence of IABD and cerebral SVDs and the total SVD score were checked using multivariate binary logistic regression (for the presence of each cerebral SVD) and linear regression (for the total SVD score as the dependent variable) after entering sex, age, and variables with $p<0.1$ in the univariate analysis. Due to multicollinearity, the presence of each cerebral SVD was analyzed separately. A two-tailed $p$ value of $<0.05$ was defined as statistically significant. There was no statistical interaction for the existence of cerebral SVDs between IABD (IASBD and $\mathrm{IADBD}$ ) and baPWV. Among the demographics and risk factors, there was no multicollinearity for the presence of each cerebral SVD and the total SVD score as the dependent variable (variance inflation factor $<2.0$ ). For the sensitivity analysis, we performed further multivariate analyses for $\mathrm{IABD} \geq 5 \mathrm{~mm} \mathrm{Hg}$ IABD $\geq 15 \mathrm{~mm} \mathrm{Hg}$, per $1-\mathrm{mm} \mathrm{Hg}$ change in IABD, both IASBD $\geq 5 \mathrm{~mm} \mathrm{Hg}$ and IADBD $\geq 5$ $\mathrm{mm} \mathrm{Hg}$, and both IASBD $\geq 10 \mathrm{~mm} \mathrm{Hg}$ and IADBD $\geq 10 \mathrm{~mm}$ $\mathrm{Hg}$ as dependent variables.

\section{RESULTS}

\section{Demographics and comparisons between patients with $\mathrm{IABD} \geq \mathbf{1 0} \mathrm{mm} \mathrm{Hg}$ and $<10 \mathrm{~mm} \mathrm{Hg}$}

The demographics and the frequency of vascular risk factors did not differ between the patients included in and excluded from this study, except for age (Supplementary Table 1 in the online-only Data Supplement). Among all included patients, $61.6 \%(742 / 1,205)$ were men and they were aged $64.6 \pm$ 11.3 years. The following characteristics were more common in the IASBD $\geq 10 \mathrm{~mm} \mathrm{Hg}$ group $(n=126,10.4 \%)$ than in the IASBD $<10 \mathrm{~mm} \mathrm{Hg}$ group $(n=1,079,89.6 \%)$ : male sex, older age, hypertension, smoking, metabolic syndrome, left ventricular hypertrophy, increased baPWV, presence of high-grade white-matter hyperintensities (HWHs), HPVSs, ALIs, and total SVD score of 1-4. Previous antithrombotic medication prior to admission was less common in the IASBD $\geq 10 \mathrm{~mm}$ Hg group (Table 1).

The following characteristics were more common in the IADBD $\geq 10 \mathrm{~mm}$ Hg group $(n=62,5.1 \%)$ than in the IADBD $<10 \mathrm{~mm} \mathrm{Hg}$ group ( $n=1,143,94.9 \%)$ : left ventricular hypertrophy, presence of HWHs and ALIs, and total SVD score of 1-3. Previous antithrombotic and antihypertensive medication before admission were less common in the IADBD $\geq 10 \mathrm{~mm}$ Hg group (Table 1).

\section{Association between IABD and presence of cerebral SVDs}

HWHs, high-grade deep WMHs, and high-grade periventricular WMHs were present in 378 (31.4\%), 288 (23.9\%), and $350(29.0 \%)$ of the 1,205 subjects, respectively. CMBs were found in 395 (32.8\%) of the patients: $29.1 \%$ in the mixed (lobar+nonlobar) area and $3.7 \%$ in the lobar area only. HPVSs and ALIs were evident in $244(20.2 \%)$ and $376(31.2 \%)$ of the patients, respectively. The total SVD scores were $0,1,2,3$, and 4 in 514 (42.7\%), 274 (22.7\%), 217 (18.0\%), 115 (9.5\%), and $85(7.1 \%)$ of the patients, respectively (Table 2 ). The comparison results including demographics, risk factors, stroke subtypes, ABI parameters, IABD ( $\geq 5 \mathrm{~mm} \mathrm{Hg}, \geq 10 \mathrm{~mm} \mathrm{Hg}$, $\geq 15 \mathrm{~mm} \mathrm{Hg}$ ), and both IASBD and IASBD $\geq 5 \mathrm{~mm} \mathrm{Hg}$ and $\geq 10 \mathrm{~mm} \mathrm{Hg}$ according to the presence or absence of each cerebral SVD are presented in Table 2 and Supplementary Table 2 (in the online-only Data Supplement).

After adjusting for sex, age, and variables with $p<0.1$ in the univariate analysis, IASBD $\geq 10 \mathrm{~mm} \mathrm{Hg}$ was independently related to the existence of HWHs [odds ratio $(\mathrm{OR})=1.94$, $95 \% \mathrm{CI}=1.32-2.84, p=0.011)$ and had a tendency to be associated with the presence of HPVSs (OR=1.45, 95\% CI $=0.49-$ $2.23, p=0.089)$ and ALIs $(\mathrm{OR}=1.42,95 \% \mathrm{CI}=0.96-2.11, p=$ 0.052). However, IASBD $\geq 10 \mathrm{~mm} \mathrm{Hg}$ was not associated with the presence of CMBs $(\mathrm{OR}=1.09,95 \% \mathrm{CI}=0.73-1.61, p=$ $0.634)$ (Table 3). The results of the sensitivity analysis of different IABD cutoff values ( $\geq 5 \mathrm{~mm} \mathrm{Hg}$ or $\geq 15 \mathrm{~mm} \mathrm{Hg}$ ) are presented in Table 3 and Supplementary Tables 3 and 4 (in the online-only Data Supplement).

IADBD $\geq 10 \mathrm{~mm} \mathrm{Hg}$ was independently related to the existence of HWHs $(\mathrm{OR}=2.23,95 \% \mathrm{CI}=1.32-3.76, p=0.012)$ and ALIs (OR=1.92, 95\% CI=1.13-3.25, $p=0.044)$, but not to CMBs and HPVSs. Moreover, both IASBD $\geq 10 \mathrm{~mm} \mathrm{Hg}$ and $\mathrm{IADBD} \geq 10 \mathrm{~mm} \mathrm{Hg}$ were also associated with the presence of HWHs and ALIs (Table 3).

\section{Association between IABD and burden of cerebral SVDs}

In multivariate linear regression with the total SVD score as the dependent variable and with adjustment for sex, age, and variables with $p<0.1$ in the univariate analysis (hypertension, diabetes mellitus, hypercholesterolemia, metabolic syndrome, alcohol intake, left ventricular hypertrophy, and baPWV), IASBD $\geq 10 \mathrm{~mm} \mathrm{Hg}(\beta=0.080, p=0.006)$ and IADBD $\geq 10$ $\mathrm{mm} \mathrm{Hg}(\beta=0.065, p=0.023)$ were significantly and positively correlated with the total SVD score (Table 4 ).

Predictability of IABD for presence of cerebral SVDs In ROC curve analyses, the AUC for IABD $\geq 10 \mathrm{~mm}$ Hg was significant for the presence of HWHs (AUC $=0.777$ for IASBD 
Table 1. Clinical characteristics and comparison of study patients according to different values of the IASBD and the IADBD

\begin{tabular}{|c|c|c|c|c|c|c|c|}
\hline & $\begin{array}{c}\text { Total } \\
(n=1,205)\end{array}$ & $\begin{array}{c}\text { IASBD } \\
<10 \mathrm{~mm} \mathrm{Hg} \\
(n=1,079)\end{array}$ & $\begin{array}{c}\text { IASBD } \\
\geq 10 \mathrm{~mm} \mathrm{Hg} \\
(n=126)\end{array}$ & $p$ & $\begin{array}{c}\text { IADBD } \\
<10 \mathrm{~mm} \mathrm{Hg} \\
(n=1,143)\end{array}$ & $\begin{array}{c}\text { IADBD } \\
\geq 10 \mathrm{~mm} \mathrm{Hg} \\
(n=62)\end{array}$ & $p$ \\
\hline \multicolumn{8}{|l|}{ Demographics } \\
\hline Sex, male & $742(61.6)$ & $652(60.4)$ & $90(71.4)$ & 0.016 & 701 (61.3) & $41(66.1)$ & 0.449 \\
\hline Age, years & $64.6 \pm 11.3$ & $64.4 \pm 11.6$ & $66.1 \pm 8.8$ & 0.049 & $64.6 \pm 11.5$ & $64.4 \pm 7.8$ & 0.258 \\
\hline \multicolumn{8}{|l|}{ Risk factors } \\
\hline Hypertension & $816(67.7)$ & 719 (66.6) & $97(77.0)$ & 0.019 & $773(67.6)$ & $43(69.4)$ & 0.777 \\
\hline Diabetes mellitus & $389(32.3)$ & 344 (31.9) & $45(35.7)$ & 0.384 & $389(33.4)$ & $19(30.6)$ & 0.651 \\
\hline Hypercholesterolemia & $231(19.2)$ & $212(19.6)$ & $19(15.1)$ & 0.218 & $216(18.9)$ & $15(24.2)$ & 0.302 \\
\hline Smoking & $364(30.2)$ & $315(29.2)$ & 49 (38.9) & 0.025 & $323(28.3)$ & $20(32.3)$ & 0.497 \\
\hline Coronary artery disease & $248(20.6)$ & $222(20.6)$ & $26(20.6)$ & 0.987 & $240(21.0)$ & $8(12.9)$ & 0.125 \\
\hline Metabolic syndrome & $531(44.1)$ & $460(42.6)$ & $71(56.3)$ & 0.003 & $482(42.4)$ & $21(33.9)$ & 0.197 \\
\hline Alcohol intake & $161(13.4)$ & $138(12.8)$ & $23(18.3)$ & 0.088 & $151(13.2)$ & $10(16.1)$ & 0.511 \\
\hline Left ventricular hypertrophy & $156(12.9)$ & $129(12.0)$ & $27(21.4)$ & 0.003 & $123(10.8)$ & $18(29.0)$ & 0.001 \\
\hline Body mass index, $\mathrm{kg} / \mathrm{m}^{2}$ & $24.0 \pm 3.08$ & $24.0 \pm 3.0$ & $24.2 \pm 3.3$ & 0.374 & $24.0 \pm 3.0$ & $23.7 \pm 3.6$ & 0.527 \\
\hline Antihypertensive medication before $A B I$ examination & $131(10.9)$ & $112(10.4)$ & $19(15.1)$ & 0.129 & $126(11.0)$ & $5(8.1)$ & 0.466 \\
\hline Thrombolytic therapy & $113(9.4)$ & $96(8.9)$ & $17(13.5)$ & 0.105 & $109(9.5)$ & $4(6.5)$ & 0.652 \\
\hline NIHSS score & $4.0 \pm 4.6$ & $4.0 \pm 4.5$ & $4.1 \pm 5.1$ & 0.729 & $4.0 \pm 4.5$ & $4.2 \pm 5.7$ & 0.745 \\
\hline Stroke subtype & & & & 0.364 & & & 0.662 \\
\hline Large-artery atherosclerosis & $410(34.0)$ & $361(33.5)$ & $49(38.9)$ & & $393(34.4)$ & $17(27.4)$ & \\
\hline Lacunar & $320(26.6)$ & $294(27.2)$ & $26(20.6)$ & & $302(26.4)$ & $18(29.0)$ & \\
\hline Undetermined negative & $386(32.0)$ & $346(32.1)$ & $40(31.7)$ & & $363(31.8)$ & $23(37.1)$ & \\
\hline Undetermined two of more causes identified & $89(7.4)$ & $78(7.2)$ & $11(8.7)$ & & $85(7.4)$ & $4(6.5)$ & \\
\hline \multicolumn{8}{|l|}{ Previous medication before admission } \\
\hline Antithrombotic medication & $219(18.2)$ & $205(19.0)$ & $14(11.1)$ & 0.028 & $215(18.8)$ & $4(6.5)$ & 0.011 \\
\hline Antihypertensive medication & $265(22.0)$ & $234(21.7)$ & $31(24.6)$ & 0.455 & $198(17.3)$ & $4(6.5)$ & 0.023 \\
\hline Lipid-lowering agents & $202(16.8)$ & $188(17.4)$ & $14(11.1)$ & 0.073 & $253(22.1)$ & $12(19.4)$ & 0.607 \\
\hline \multicolumn{8}{|l|}{$\mathrm{ABI}$ parameters } \\
\hline Pulse rate, per minute & $69.9 \pm 13.2$ & $69.6 \pm 13.4$ & $74.1 \pm 8.6$ & 0.357 & $70.0 \pm 12.9$ & $67.4 \pm 20.1$ & 0.662 \\
\hline Arm SBP, mm Hg & $149.8 \pm 22.4$ & $149.7 \pm 22.3$ & $151.3 \pm 23.3$ & 0.427 & $150.1 \pm 22.4$ & $144.9 \pm 21.7$ & 0.073 \\
\hline Arm DBP, mm Hg & $85.4 \pm 12.4$ & $85.2 \pm 12.5$ & $86.5 \pm 11.9$ & 0.293 & $85.5 \pm 12.5$ & $82.4 \pm 10.5$ & 0.055 \\
\hline baPWV, m/s & $19.8 \pm 4.9$ & $19.7 \pm 5.0$ & $20.7 \pm 4.4$ & 0.041 & $19.8 \pm 5.0$ & $19.9 \pm 4.6$ & 0.909 \\
\hline \multicolumn{8}{|l|}{ Cerebral SVDs } \\
\hline HWHs & $378(31.4)$ & $320(29.7)$ & $58(46.0)$ & 0.001 & $348(30.4)$ & $30(48.4)$ & 0.005 \\
\hline Deep white matter & $288(23.9)$ & $246(22.8)$ & $42(33.3)$ & 0.011 & $266(23.3)$ & $22(35.5)$ & 0.033 \\
\hline Periventricular white matter & $350(29.0)$ & $308(28.5)$ & $42(33.0)$ & 0.263 & 327 (28.6) & $23(37.1)$ & 0.152 \\
\hline Presence of CMBs & $395(32.8)$ & $350(32.4)$ & $45(35.7)$ & 0.458 & $192(16.8)$ & $12(19.4)$ & 0.601 \\
\hline Location of CMBs & & & & 0.665 & & & 0.289 \\
\hline No CMBs & $810(67.2)$ & $729(67.6)$ & $81(64.3)$ & & $767(67.1)$ & $43(69.4)$ & \\
\hline Mixed (lobar+nonlobar) & $351(29.1)$ & $312(28.9)$ & 39 (31.0) & & $332(29.0)$ & $19(30.6)$ & \\
\hline Lobar only & $44(3.7)$ & $38(3.5)$ & $6(4.8)$ & & $44(3.8)$ & $0(0.0)$ & \\
\hline HPVSS & $244(20.2)$ & $210(19.5)$ & $34(27.0)$ & 0.047 & 227 (19.9) & $17(27.4)$ & 0.147 \\
\hline ALls & $376(31.2)$ & $326(30.2)$ & $50(39.7)$ & 0.033 & $348(30.4)$ & $28(45.2)$ & 0.023 \\
\hline Total SVD score & & & & 0.004 & & & 0.032 \\
\hline 0 & $514(42.7)$ & $479(44.4)$ & $35(27.8)$ & & $498(43.6)$ & $16(25.8)$ & \\
\hline 1 & $274(22.7)$ & $242(22.4)$ & $32(25.4)$ & & $259(22.7)$ & $15(24.2)$ & \\
\hline 2 & $217(18.0)$ & $186(17.2)$ & $31(24.6)$ & & $199(17.4)$ & $18(29.0)$ & \\
\hline 3 & $115(9.5)$ & $96(8.9)$ & $19(15.1)$ & & $106(9.3)$ & $9(14.5)$ & \\
\hline 4 & $85(7.1)$ & $76(7.0)$ & $9(7.1)$ & & $81(7.1)$ & $4(6.5)$ & \\
\hline
\end{tabular}

Data are $n(\%)$ or mean \pm SD values.

ABI: ankle-brachial index, ALIs: asymptomatic lacunar infarctions, baPWV: brachial-ankle pulse wave velocity, CMBs: cerebral microbleeds, DBP: diastolic blood pressure, HPVSs: high-grade perivascular spaces, HWHs: high-grade white-matter hyperintensities, IADBD: interarm diastolic blood pressure difference, IASBD: interarm systolic blood pressure difference, NIHSS: National Institutes of Health Stroke Scale, SBP: systolic blood pressure, SVD: small-vessel disease. 
Table 2. Clinical characteristics and comparison of the study patients according to the presence of different types of cerebral SVDs

\begin{tabular}{|c|c|c|c|c|c|c|c|c|}
\hline & $\begin{array}{l}\text { HWHs }(-) \\
(n=827)\end{array}$ & $\begin{array}{l}\text { HWHs }(+) \\
(n=378)\end{array}$ & $\begin{array}{l}\text { CMBs }(-) \\
(n=810)\end{array}$ & $\begin{array}{l}\text { CMBs }(+) \\
(n=395)\end{array}$ & $\begin{array}{l}\text { HPVSs }(-) \\
(n=961)\end{array}$ & $\begin{array}{l}\text { HPVSs }(+) \\
(n=244)\end{array}$ & $\begin{array}{l}\text { ALIs }(-) \\
(n=829)\end{array}$ & $\begin{array}{l}\text { ALIs (+) } \\
(n=376)\end{array}$ \\
\hline \multicolumn{9}{|l|}{ Demographics } \\
\hline Sex, male & $522(63.1)$ & $220(58.2)$ & 509 (62.8) & $233(59.0)$ & 597 (62.1) & $145(59.4)$ & $530(63.9)$ & $212(56.4)^{*}$ \\
\hline Age, years & $64.1 \pm 11.3$ & $65.8 \pm 11.4^{*}$ & $64.5 \pm 11.5$ & $64.8 \pm 11.1$ & $64.4 \pm 11.5$ & $65.6 \pm 10.8^{+}$ & $64.2 \pm 11.4$ & $65.4 \pm 11.1$ \\
\hline \multicolumn{9}{|l|}{ Risk factors } \\
\hline Hypertension & $520(62.9)$ & $296(78.3)^{*}$ & $545(67.3)$ & $271(68.6)$ & $639(66.5)$ & $177(72.5) \dagger$ & $538(64.9)$ & $278(73.9)^{*}$ \\
\hline Diabetes mellitus & $254(30.7)$ & $135(35.7)^{+}$ & $274(33.8)$ & $115(29.1)$ & $303(31.5)$ & $86(35.2)$ & $254(30.6)$ & $135(35.9)^{+}$ \\
\hline Hypercholesterolemia & $153(18.5)$ & 78 (20.6) & $170(21.0)$ & $61(15.4)^{*}$ & $177(18.4)$ & $54(22.1)$ & $153(18.5)$ & $78(20.7)$ \\
\hline Smoking & $251(30.4)$ & $113(29.9)$ & $233(28.8)$ & 131 (33.2) & $281(29.2)$ & $83(34.0)$ & 257 (31.0) & $107(28.5)$ \\
\hline Coronary artery disease & $174(20.9)$ & 74 (19.9) & $177(21.9)$ & 71 (18.0) & $205(21.3)$ & $43(17.6)$ & $170(20.5)$ & $78(20.7)$ \\
\hline Metabolic syndrome & $346(41.8)$ & $185(48.9)^{*}$ & $358(44.2)$ & $173(43.8)$ & $418(43.5)$ & $113(46.3)$ & $339(40.9)$ & $192(51.1)^{*}$ \\
\hline Alcohol intake & $108(13.1)$ & $53(14.0)$ & $113(14.0)$ & 48 (12.2) & $130(13.5)$ & $31(12.7)$ & $98(11.8)$ & $63(16.8)^{*}$ \\
\hline Left ventricular hypertrophy & $107(12.9)$ & $49(13.0)$ & $195(11.7)$ & $61(15.4)^{+}$ & $115(12.0)$ & $41(16.8)^{*}$ & $111(13.4)$ & $45(12.0)$ \\
\hline Body mass index, $\mathrm{kg} / \mathrm{m}^{2}$ & $24.0 \pm 3.0$ & $24.0 \pm 3.2$ & $24.0 \pm 3.0$ & $23.9 \pm 3.0$ & $23.9 \pm 3.0$ & $24.2 \pm 3.3$ & $23.9 \pm 3.0$ & $24.1 \pm 3.2$ \\
\hline $\begin{array}{l}\text { Antihypertensive medication before } A B I \\
\text { examination }\end{array}$ & $92(11.1)$ & 39 (10.3) & $78(9.6)$ & $53(13.4)^{*}$ & $102(10.6)$ & $29(11.9)$ & $89(10.7)$ & $42(11.2)$ \\
\hline Thrombolytic therapy & $82(9.9)$ & $31(8.2)$ & $69(8.5)$ & $44(11.1)$ & $88(9.2)$ & $25(10.2)$ & $79(9.5)$ & $34(9.0)$ \\
\hline NIHSS score & $3.8 \pm 4.4$ & $4.4 \pm 4.9^{+}$ & $4.0 \pm 4.7$ & $4.0 \pm 4.4$ & $3.9 \pm 4.5$ & $4.4 \pm 4.6$ & $4.0 \pm 4.5$ & $4.0 \pm 4.7$ \\
\hline \multicolumn{9}{|l|}{ Stroke subtype } \\
\hline Large-artery atherosclerosis & $286(34.6)$ & $124(32.8)$ & $281(34.7)$ & $129(32.7)$ & $312(32.5)$ & $98(40.2)$ & $278(33.5)$ & $132(35.1)$ \\
\hline Lacunar & $217(26.2)$ & $103(27.2)$ & $210(25.9)$ & $110(27.8)$ & $256(26.6)$ & 64 (26.2) & $221(26.7)$ & $99(26.3)$ \\
\hline Undetermined negative & $255(30.8)$ & $131(34.7)$ & $264(32.6)$ & $122(30.9)$ & $318(33.1)$ & $68(27.9)$ & $263(31.7)$ & $123(32.7)$ \\
\hline Undetermined two or more causes identified & $69(8.3)$ & $20(5.3)$ & $55(6.8)$ & $34(8.6)$ & $75(7.8)$ & $14(5.7)$ & $67(8.1)$ & $22(5.9)$ \\
\hline \multicolumn{9}{|l|}{ Previous medication before admission } \\
\hline Antithrombotic medication & $155(18.7)$ & $64(16.9)$ & $156(19.3)$ & $63(15.9)$ & $177(18.4)$ & $42(17.2)$ & $156(18.8)$ & $63(16.8)$ \\
\hline Antihypertensive medication & $190(23.0)$ & 75 (19.8) & $173(21.4)$ & $92(22.3)$ & $214(22.3)$ & $51(20.9)$ & $185(22.3)$ & $80(21.3)$ \\
\hline Lipid-lowering agents & $136(16.4)$ & $66(17.5)$ & $135(16.7)$ & $67(17.0)$ & $159(16.5)$ & $43(17.6)$ & $138(16.6)$ & $64(17.0)$ \\
\hline \multicolumn{9}{|l|}{$\mathrm{ABI}$ parameters } \\
\hline Pulse rate, per minute & $69.5 \pm 12.2$ & $72.3 \pm 17.6$ & $69.5 \pm 12.1$ & $71.1 \pm 16.4$ & $69.8 \pm 12.3$ & $71.0 \pm 19.0$ & $70.2 \pm 12.8$ & $68.5 \pm 15.1$ \\
\hline Arm SBP, mm Hg & $149.9 \pm 23.0$ & $149.9 \pm 21.0$ & $149.5 \pm 22.0$ & $150.6 \pm 23.7$ & $149.5 \pm 22.3$ & $151.2 \pm 22.6$ & $149.4 \pm 23.0$ & $150.8 \pm 21.1$ \\
\hline Arm DBP, $\mathrm{mm} \mathrm{Hg}$ & $85.4 \pm 12.7$ & $85.4 \pm 11.8$ & $85.3 \pm 12.2$ & $85.6 \pm 12.8$ & $85.4 \pm 12.4$ & $85.3 \pm 12.4$ & $85.2 \pm 12.6$ & $85.7 \pm 11.9$ \\
\hline baPWV, $\mathrm{m} / \mathrm{s}$ & $19.7 \pm 4.9$ & $21.0 \pm 4.9^{*}$ & $19.8 \pm 5.0$ & $21.7 \pm 4.8^{*}$ & $19.9 \pm 3.2$ & $20.8 \pm 5.0+$ & $19.7 \pm 5.0$ & $21.1 \pm 4.9^{*}$ \\
\hline \multicolumn{9}{|l|}{ IABD, mm Hg } \\
\hline \multicolumn{9}{|l|}{ IASBD } \\
\hline$\geq 5$ & $309(37.4)$ & $167(44.2)^{*}$ & $341(42.1)$ & $135(34.2)^{*}$ & $375(39.0)$ & $101(41.4)$ & $321(38.7)$ & $155(41.2)$ \\
\hline$\geq 10$ & $68(8.2)$ & $58(15.3)^{*}$ & $81(10.0)$ & $45(11.4)$ & $92(9.6)$ & $34(13.9)^{*}$ & $76(9.2)$ & $50(13.3)^{*}$ \\
\hline$\geq 15$ & $12(1.5)$ & $19(5.0)^{*}$ & $20(2.5)$ & $11(2.8)$ & $19(2.0)$ & $12(4.9)^{*}$ & $15(1.8)$ & $16(4.3)^{*}$ \\
\hline \multicolumn{9}{|l|}{ IADBD } \\
\hline$\geq 5$ & $212(25.6)$ & $120(31.7)^{*}$ & $231(28.5)$ & $101(25.6)$ & $268(27.9)$ & $64(26.2)$ & $214(25.8)$ & $118(31.4)^{*}$ \\
\hline$\geq 10$ & $32(3.9)$ & $30(7.9)^{*}$ & $43(5.3)$ & $19(4.8)$ & $45(4.7)$ & $17(7.0)$ & $34(4.1)$ & $28(7.4)^{*}$ \\
\hline$\geq 15$ & $11(1.3)$ & $13(3.4)^{*}$ & $18(2.2)$ & $6(1.5)$ & $16(1.7)$ & $8(3.3)$ & $12(1.4)$ & $12(3.2)^{*}$ \\
\hline IASBD and IADBD $\geq 5 \mathrm{~mm} \mathrm{Hg}$ & 115 (13.9) & $74(19.6)^{*}$ & $143(17.7)$ & $46(11.6)^{*}$ & $148(15.4)$ & $41(16.8)$ & $120(14.5)$ & $69(18.4)^{+}$ \\
\hline IASBD and IADBD $\geq 10 \mathrm{~mm} \mathrm{Hg}$ & $14(1.7)$ & $16(4.2)^{*}$ & $21(2.6)$ & $9(2.3)$ & $19(2.0)$ & $11(4.5)^{*}$ & $16(1.9)$ & $14(3.7)^{+}$ \\
\hline
\end{tabular}

Data are $n(\%)$ or mean \pm SD values.

${ }^{*} p<0.05,{ }^{\dagger} p<0.1$.

ABI: ankle-brachial index, ALls: asymptomatic lacunar infarctions, baPWV: brachial-ankle pulse wave velocity, CMBs: cerebral microbleeds, DBP: diastolic blood pressure, HPVSs: high-grade perivascular spaces, HWHs: high-grade white-matter hyperintensities, IABD: interarm blood pressure difference, IADBD: interarm diastolic blood pressure difference, IASBD: interarm systolic blood pressure difference, NIHSS: National Institutes of Health Stroke Scale, SBP: systolic blood pressure, SVDs: small-vessel diseases. 
Table 3. Results of the multivariate analysis for the presence of cerebral SVDs according to IASBD and IADBD

\begin{tabular}{|c|c|c|c|c|}
\hline IABD & HWHs (a) & CMBs (b) & HPVSs (c) & ALIs (d) \\
\hline \multicolumn{5}{|l|}{ IASBD (mm Hg) } \\
\hline$\geq 5$ & $1.22(0.94-1.57)$ & $0.68(0.53-0.88)^{*}$ & $1.05(0.78-1.40)$ & $1.03(0.80-1.40)$ \\
\hline$\geq 10$ & $1.94(1.32-2.84)^{*}$ & $1.09(0.73-1.61)$ & $1.45(0.49-2.23)^{+}$ & $1.42(0.96-2.11)^{+}$ \\
\hline$\geq 15$ & $3.37(1.58-7.11)^{*}$ & $1.02(0.47-2.19)$ & $2.23(1.04-4.75)^{*}$ & $2.12(1.02-4.41)^{*}$ \\
\hline Per 1 increase & $1.05(1.02-1.08)^{*}$ & $0.99(0.97-1.01)$ & $1.00(0.98-1.02)$ & $1.02(1.01-1.08)^{*}$ \\
\hline \multicolumn{5}{|l|}{ IADBD (mm Hg) } \\
\hline$\geq 5$ & $1.39(1.06-1.83)^{*}$ & $0.85(0.64-1.12)$ & $0.91(0.66-1.25)$ & $1.30(0.99-1.71)^{+}$ \\
\hline$\geq 10$ & $2.23(1.32-3.76)^{*}$ & $0.90(0.51-1.58)$ & $1.48(0.82-2.64)$ & $1.92(1.13-3.25)^{*}$ \\
\hline$\geq 15$ & $3.06(1.34-7.02)^{*}$ & $0.69(0.26-1.76)$ & $1.97(0.82-4.72)$ & $1.30(0.99-1.71)^{+}$ \\
\hline Per 1 increase & $1.06(1.03-1.10)^{*}$ & $0.98(0.94-1.01)$ & $1.01(0.97-1.05)$ & $1.05(1.02-1.09)^{*}$ \\
\hline IASBD and IADBD $\geq 5 \mathrm{~mm} \mathrm{Hg}$ & $1.48(1.06-2.06)^{*}$ & $0.59(0.41-1.25)$ & $1.06(0.72-1.56)$ & $1.29(0.92-1.80)$ \\
\hline IASBD and IADBD $\geq 10 \mathrm{~mm} \mathrm{Hg}$ & $2.63(1.25-5.54)^{*}$ & $0.82(0.37-1.84)$ & $2.17(1.01-4.69)^{*}$ & $1.89(0.90-3.99)^{+}$ \\
\hline
\end{tabular}

Data are odds ratio $(95 \% \mathrm{Cl})$ values. Adjusted for sex, age, hypertension, diabetes mellitus, metabolic syndrome, and baPWV (a), adjusted for sex, age, hypercholesterolemia, left ventricular hypertrophy, antihypertensive medication after admission, and baPWV (b), adjusted for sex, age, hypertension, left ventricular hypertrophy, and baPWV (c), and adjusted for sex, age, hypertension, diabetes mellitus, metabolic syndrome, alcohol intake, and baPWV (d).

${ }^{*} p<0.05,{ }^{+} p<0.1$.

ALls: asymptomatic lacunar infarctions, baPWV: brachial-ankle pulse wave velocity, CMBs: cerebral microbleeds, HPVSs: high-grade perivascular spaces, HWHs: high-grade white-matter hyperintensities, IABD: interarm blood pressure difference, IADBD: interarm diastolic blood pressure difference, IASBD: interarm systolic blood pressure difference, SVDs: small-vessel diseases.

Table 4. Association of IABD with the total SVD score

\begin{tabular}{|c|c|c|c|c|c|c|}
\hline & \multicolumn{2}{|c|}{ Nonstandardized coefficients } & \multirow{2}{*}{ Standardized coefficient $(\beta)$} & \multirow[b]{2}{*}{$t$} & \multirow{2}{*}{$p^{*}$} & \multirow{2}{*}{$\mathrm{R}^{2}$} \\
\hline & B & SE & & & & \\
\hline \multicolumn{7}{|l|}{ IASBD $(\mathrm{mm} \mathrm{Hg})$} \\
\hline$\geq 5$ & -0.022 & 0.076 & -0.009 & -0.296 & 0.768 & 0.020 \\
\hline$\geq 10$ & 0.330 & 0.120 & 0.080 & 2.759 & 0.006 & 0.027 \\
\hline$\geq 15$ & 0.644 & 0.232 & 0.081 & 2.772 & 0.006 & 0.027 \\
\hline Per 1 increase & 0.013 & 0.006 & 0.064 & 2.167 & 0.030 & 0.024 \\
\hline \multicolumn{7}{|l|}{ IADBD (mm Hg) } \\
\hline$\geq 5$ & 0.076 & 0.082 & 0.027 & 0.926 & 0.355 & 0.021 \\
\hline$\geq 10$ & 0.374 & 0.164 & 0.065 & 2.281 & 0.023 & 0.025 \\
\hline$\geq 15$ & 0.517 & 0.259 & 0.057 & 1.992 & 0.047 & 0.024 \\
\hline Per 1 increase & 0.024 & 0.010 & 0.066 & 2.306 & 0.021 & 0.025 \\
\hline IASBD and IADBD $\geq 5 \mathrm{~mm} \mathrm{Hg}$ & 0.045 & 0.101 & 0.013 & 0.444 & 0.657 & 0.020 \\
\hline IASBD and IADBD $\geq 10 \mathrm{~mm} \mathrm{Hg}$ & 0.492 & 0.233 & 0.061 & 2.107 & 0.035 & 0.024 \\
\hline
\end{tabular}

*Results from multivariate linear regression with total SVD score as the dependent variable and with adjustment for sex, age and variables with $p<0.1$ in the univariate analysis (hypertension, diabetes mellitus, hypercholesterolemia, metabolic syndrome, alcohol intake, left ventricular hypertrophy, and baPWV).

baPWV: brachial-ankle pulse wave velocity, IABD: interarm blood pressure difference, IADBD: interarm diastolic blood pressure difference, IASBD: interarm systolic blood pressure difference, SE: standard error, SVDs: small-vessel diseases.

$\geq 10 \mathrm{~mm} \mathrm{Hg}$ and 0.736 for IADBD $\geq 10 \mathrm{~mm} \mathrm{Hg}$ ) and ALIs (AUC $=0.745$ for IASBD $\geq 10 \mathrm{~mm} \mathrm{Hg}$ and 0.751 for IADBD $\geq 10 \mathrm{~mm} \mathrm{Hg}$ ), but not for CMBs and HPVSs (Supplementary Table 5 in the online-only Data Supplement).

\section{DISCUSSION}

This study found that IASBD $\geq 10 \mathrm{~mm} \mathrm{Hg}$ was independently related to the existence of HWHs and had a tenden- cy to be related to the presence of HPVSs and ALIs. IADBD $\geq 10 \mathrm{~mm} \mathrm{Hg}$ was also associated with the existence of HWHs and ALIs, but not with CMBs and HPVSs. Furthermore, IASBD $\geq 10 \mathrm{~mm} \mathrm{Hg}$ and/or IADBD $\geq 10 \mathrm{~mm}$ Hg were positively correlated with the burden of cerebral SVDs. Thus, our study has revealed that IASBD and IADBD are variously related to the presence and burden of cerebral SVDs. In the recent Framingham Heart Study, a high IABD was associated with an increased risk of Alzheimer's disease and subclinical 
brain injury. ${ }^{27}$ The results of the present study are in line with those of that previous population-based study, and they provide additional information on stroke subjects. In clinical practice it is easier to measure IABD than to perform brain CT or MRI, and so our findings suggest the usefulness of IABD as one of the screening tools or criterion for investigating the presence of cerebral SVDs.

The mechanisms underlying the present results are unclear, but following hypotheses can be proposed. First, a large IABD can result from stenosis of the proximal aorta, brachiocephalic artery, and subclavian artery. ${ }^{3}$ This would mean that the large IABD is associated with poor cerebral perfusion, which results in damage to the brain parenchyma. ${ }^{11}$ A previous study found cerebral hypoperfusion to be associated with an increased cerebral SVD burden. ${ }^{28}$ Second, increased arterial stiffness would be an associated factor. IASBD $>10$ $\mathrm{mm} \mathrm{Hg}$ was previously found to be related to increased arterial stiffness, ${ }^{12}$ and it is well known that this is associated with the presence of cerebral SVDs. ${ }^{15}$ In the present study, baPWV was higher in patients with IASBD $\geq 10 \mathrm{~mm}$ Hg. Third, the well-known cardiovascular risk factors of hypertension and diabetes mellitus are also associated with cerebral SVDs. Therefore, the relationship between IABD and cerebral SVDs elucidated in the present study might be an epiphenomenon elicited by similar associated factors.

Our study revealed that $\mathrm{IABD} \geq 10 \mathrm{~mm} \mathrm{Hg}$ was related to the existence of HWHs, but was not associated with the presence of CMBs and HPVSs. Both CMBs and HPVSs are significantly associated with arterial stiffness, ${ }^{15}$ impaired permeability of the blood-brain barrier, and vascular inflammation, all of which are factors closely related to HWHs. ${ }^{5,89}$ Accordingly, because IABD is also related to increased arterial stiffness, it is likely that not only HMHs but also CMBs and HPVSs may be associated with IABD, which contrasts with the results of the present study. In contrast to cerebral hypoperfusion resulting from an IABD, CMBs can be induced by hypertension-related mechanical damage that ruptures tight junctions. ${ }^{9}$ Therefore, this difference in the mechanism resulting in the development of CMBs can explain the discrepancy of the nonsignificant relationship between IABD and the presence of CMBs. Also, the reasons for the lack of an association between IABD and HPVSs in this study remain unknown. Because IASBD $\geq 15 \mathrm{~mm} \mathrm{Hg}$ was found to be associated with HPVSs, our results might have been due to a weak statistically relationship between IABD $\geq 10 \mathrm{~mm} \mathrm{Hg}$ and HPVSs or decreased statistical power resulting from controlling for other strongly associated factors such as age and hypertension.

The present study further found that IASBD was significantly associated with deep WMHs, while not being associated with periventricular WMHs. A previous study investigating the correlation between brain MRI and brain histopathology found that periventricular WMHs were mainly associated with myelin loss or subependymal gliosis, which represent senile changes, whereas deep WMHs were associated with the loss of myelin or subependymal gliosis as well as ischemic damage of vascular origin. ${ }^{29}$ Although the present study did not reveal the exact mechanism, our findings suggest that $\mathrm{IABD}$ is at least partially associated with or contributes to cerebral SVDs via cerebral ischemia (HWHs, HPVSs, and ALIs) rather than cerebral hemorrhage (CMBs).

This study was subject to some limitations. First, although $\mathrm{ABI}$ is routinely measured in almost all consecutive patients, selection bias was possible due to the retrospective design of our study. Second, our study population was limited to noncardioembolic stroke patients. Since all stroke patients receive brain CT and/or MRI, which can give information about cerebral SVDs, adding IABD has little value when screening SVDs. However, our study is significant in that it showed a correlation between cerebral SVDs and IABD, which is easy to measure in clinical practice. Third, the characteristics of our study population make it difficult to generalize our findings to another population or cohort. Fourth, multiple, automatic, and simultaneous assessments are recommended for accurate IABD measurements, rather than one-time, manual, and sequential evaluation methods. We used an automatic and simultaneous device, but IABD was measured only once when checking $\mathrm{ABI}$, and so the consistency of the measured IABD values is uncertain. Finally, because our study had a cross-sectional design, further long-term follow-up research is needed into the association of IABD with cerebral SVDs.

In conclusion, our study suggests that IABD $\geq 10 \mathrm{~mm} \mathrm{Hg}$ could be a useful indicator of the presence and burden of cerebral SVDs in noncardioembolic stroke patients.

\section{Supplementary Materials}

The online-only Data Supplement is available with this article at https://doi.org/10.3988/jcn.2019.15.2.159.

\section{Conflicts of Interest}

The authors have no potential conflicts of interest to disclose.

\section{Acknowledgements}

This project was supported by grant from the Basic Science Research Program through the National Research Foundation of Korea funded by the Ministry of Education (2018R1D1A1B07040959).

\section{REFERENCES}

1. Clark CE, Taylor RS, Shore AC, Campbell JL. Prevalence of systolic inter-arm differences in blood pressure for different primary care populations: systematic review and meta-analysis. Br J Gen Pract 2016;66: e838-e847. 
2. Kim J, Song TJ, Song D, Lee HS, Nam CM, Nam HS, et al. Interarm blood pressure difference and mortality in patients with acute ischemic stroke. Neurology 2013;80:1457-1464.

3. Clark CE, Taylor RS, Shore AC, Ukoumunne OC, Campbell JL. Association of a difference in systolic blood pressure between arms with vascular disease and mortality: a systematic review and meta-analysis. Lancet 2012;379:905-914.

4. Aboyans V, Criqui MH, McDermott MM, Allison MA, Denenberg JO, Shadman R, et al. The vital prognosis of subclavian stenosis. J Am Coll Cardiol 2007;49:1540-1545.

5. Han F, Zhai FF, Wang Q, Zhou LX, Ni J, Yao M, et al. Prevalence and risk factors of cerebral small vessel disease in a Chinese populationbased sample. J Stroke 2018;20:239-246.

6. Song TJ, Kim J, Song D, Nam HS, Kim YD, Lee HS, et al. Association of cerebral microbleeds with mortality in stroke patients having atrial fibrillation. Neurology 2014;83:1308-1315.

7. Tsai HH, Kim JS, Jouvent E, Gurol ME. Updates on prevention of hemorrhagic and lacunar strokes. J Stroke 2018;20:167-179.

8. Doubal FN, MacLullich AM, Ferguson KJ, Dennis MS, Wardlaw JM. Enlarged perivascular spaces on MRI are a feature of cerebral small vessel disease. Stroke 2010;41:450-454

9. Kim BJ, Lee SH. Cerebral microbleeds: their associated factors, radiologic findings, and clinical implications. J Stroke 2013;15:153-163.

10. Song TJ, Kim J, Song D, Yoo J, Lee HS, Kim YJ, et al. Total cerebral small-vessel disease score is associated with mortality during followup after acute ischemic stroke. J Clin Neurol 2017;13:187-195.

11. Ochoa VM, Yeghiazarians Y. Subclavian artery stenosis: a review for the vascular medicine practitioner. Vasc Med 2011;16:29-34.

12. Canepa M, Milaneschi Y, Ameri P, AlGhatrif M, Leoncini G, Spallarossa P, et al. Relationship between inter-arm difference in systolic blood pressure and arterial stiffness in community-dwelling older adults. J Clin Hypertens (Greenwich) 2013;15:880-887.

13. Song TJ, Cho HJ, Chang Y, Choi K, Jung AR, Youn M, et al. Low plasma proportion of omega 3-polyunsaturated fatty acids predicts poor outcome in acute non-cardiogenic ischemic stroke patients. $J$ Stroke 2015;17:168-176.

14. Chang Y, Kim J, Kim MH, Kim YJ, Song TJ. Interarm blood pressure difference is associated with early neurological deterioration, poor short-term functional outcome, and mortality in noncardioembolic stroke patients. J Clin Neurol 2018;14:555-565.

15. Song TJ, Kim J, Kim YD, Nam HS, Lee HS, Nam CM, et al. The distribution of cerebral microbleeds determines their association with arterial stiffness in non-cardioembolic acute stroke patients. Eur J Neurol 2014;21:463-469.

16. Motobe K, Tomiyama H, Koji Y, Yambe M, Gulinisa Z, Arai T, et al. Cut-off value of the ankle-brachial pressure index at which the accu- racy of brachial-ankle pulse wave velocity measurement is diminished. Circ J 2005;69:55-60.

17. Adams HP Jr, Bendixen BH, Kappelle LJ, Biller J, Love BB, Gordon $\mathrm{DL}$, et al. Classification of subtype of acute ischemic stroke. Definitions for use in a multicenter clinical trial. TOAST. Trial of Org 10172 in Acute Stroke Treatment. Stroke 1993;24:35-41.

18. Yamashina A, Tomiyama H, Takeda K, Tsuda H, Arai T, Hirose K, et al. Validity, reproducibility, and clinical significance of noninvasive brachial-ankle pulse wave velocity measurement. Hypertens Res 2002;25:359364.

19. Verberk WJ, Kessels AG, Thien T. Blood pressure measurement method and inter-arm differences: a meta-analysis. Am J Hypertens 2011;24:1201-1208.

20. Moon J, Choi KH, Park JH, Song TJ, Choi YS, Kim JH, et al. Sympathetic overactivity based on heart-rate variability in patients with obstructive sleep apnea and cerebral small-vessel disease. J Clin Neurol 2018;14:310-319.

21. Song TJ, Park JH, Choi KH, Chang Y, Moon J, Kim JH, et al. Moderate-to-severe obstructive sleep apnea is associated with cerebral small vessel disease. Sleep Med 2017;30:36-42.

22. Song TJ, Kim J, Lee HS, Nam CM, Nam HS, Kim EH, et al. Differential impact of unrecognised brain infarction on stroke outcome in non-valvular atrial fibrillation. Thromb Haemost 2014;112:1312-1318.

23. Wardlaw JM, Smith EE, Biessels GJ, Cordonnier C, Fazekas F, Frayne $\mathrm{R}$, et al. Neuroimaging standards for research into small vessel disease and its contribution to ageing and neurodegeneration. Lancet Neurol 2013;12:822-838.

24. Staals J, Makin SD, Doubal FN, Dennis MS, Wardlaw JM. Stroke subtype, vascular risk factors, and total MRI brain small-vessel disease burden. Neurology 2014;83:1228-1234.

25. Song TJ, Kim YD, Yoo J, Kim J, Chang HJ, Hong GR, et al. Association between aortic atheroma and cerebral small vessel disease in patients with ischemic stroke. J Stroke 2016;18:312-320.

26. Schillaci G, Verdecchia P, Borgioni C, Ciucci A, Guerrieri M, Zampi I, et al. Improved electrocardiographic diagnosis of left ventricular hypertrophy. Am J Cardiol 1994;74:714-719.

27. Pase MP, Beiser A, Aparicio H, DeCarli C, Vasan RS, Murabito J, et al. Interarm differences in systolic blood pressure and the risk of dementia and subclinical brain injury. Alzheimers Dement 2016;12:438-445.

28. Arba F, Mair G, Carpenter T, Sakka E, Sandercock PAG, Lindley RI, et al. Cerebral white matter hypoperfusion increases with small-vessel disease burden. Data from the third international stroke trial. J Stroke Cerebrovasc Dis 2017;26:1506-1513.

29. Fazekas F, Schmidt R, Scheltens P. Pathophysiologic mechanisms in the development of age-related white matter changes of the brain. Dement Geriatr Cogn Disord 1998;9 Suppl 1:2-5. 